\title{
Statistical equilibrium in simple exchange games II. The redistribution game
}

\author{
U. Garibaldi ${ }^{1, a}$, E. Scalas ${ }^{2}$, and P. Viarengo ${ }^{3}$ \\ 1 IMEM-CNR, Physics Department, Genoa University, via Dodecaneso 33, 16146 Genoa, Italy \\ 2 Department of Advanced Sciences and Technology, Laboratory on Complex Systems, East Piedmont University, \\ Via Bellini 25 g, 15100 Alessandria, Italy \\ 3 Department of Aerospace Engineering, University of Naples "Federico II", Piazzale Tecchio 80, 80125 Naples, Italy
}

Received 30 July 2007 / Received in final form 8 October 2007

Published online 8 December 2007 - (C) EDP Sciences, Società Italiana di Fisica, Springer-Verlag 2007

\begin{abstract}
We propose a simple stochastic exchange game mimicking taxation and redistribution. There are $g$ agents and $n$ coins; taxation is modeled by randomly extracting some coins; then, these coins are redistributed to agents following Polya's scheme. The individual wealth equilibrium distribution for the resulting Markov chain is the multivariate symmetric Polya distribution. In the continuum limit, the wealth distribution converges to a Gamma distribution, whose form factor is just the initial redistribution weight. The relationship between this taxation-and-redistribution scheme and other simple conservative stochastic exchange games (such as the BDY game) is discussed.
\end{abstract}

PACS. 05.40.-a Fluctuation phenomena, random processes, noise, and Brownian motion - 89.65.Gh Economics; econophysics, financial markets, business and management - 02.50. $\mathrm{Cw}$ Probability theory 05.60.-k Transport processes

\section{Introduction}

The allocation of a finite number of objects into a finite number of categories is a classical problem in combinatorics and was used by Boltzmann at the beginning of the development of Kinetic Theory. In a seminal paper [1], Boltzmann studied the distribution of a fixed number $p$ of "energy elements" $\epsilon_{0}$ into a fixed number $n$ of molecules. In the limit $p \rightarrow \infty$, and $\epsilon_{0} \rightarrow 0$, with $p \epsilon_{0}=E_{0}=$ const, he hoped to recover Maxwell's distribution for the marginal energy of one molecule, that is $P(x)=\operatorname{Gamma}\left(\frac{3}{2}, x_{0}\right) \sim x^{1 / 2} \exp \left[-x / x_{0}\right]$ [2], where $x_{0}=\frac{E_{0}}{n}$ is the average energy per particle. However, under the hypothesis of equiprobability for all the possible states of the molecules, he missed the point, and he instead obtained $P(x)=\operatorname{Gamma}\left(1, x_{0}\right) \sim \exp \left[-x / x_{0}\right]$. Let us refer to this procedure as "Boltzmann I" method. In a subsequent paper $[3,4]$, he radically changed the model. Instead of dividing energy elements within particles, he allocated particles on energy levels. Whereas in the former approach the energy constraint was automatically satisfied, in the latter (and more fortunate) method, the condition of energy conservation must be explicitly taken into account. The celebrated formula $P\left(x_{i}\right) \sim g_{i} \exp \left[-x_{i} / x_{0}\right]$, where $g_{i}$ is the degeneracy of the $i$-th level, is derived from the most probable frequency distribution. From the

\footnotetext{
a e-mail: garibaldi@fisica.unige.it
}

nature of $\mu$-space (configuration space), Boltzmann obtained that $g_{i} \sim \sqrt{x_{i}}$ and the correct Maxwell's distribution followed in the appropriate continuum limit. We call this "Boltmann II" method.

This short historical excursus is useful to introduce the different description levels and the different probability methods already available at the very beginning of Kinetic Theory. "Boltzmann I" method (almost ignored in the literature as well as in textbooks) is based on an exact marginalization of the joint probability distribution for all molecules, whereas "Boltzmann II" method (universally known) rests on the multiplicity factor for the frequency vector. While the former approach is exact and general, the latter one is approximate and particular, being valid if and only if all joint descriptions of molecules are supposed equiprobable. Thus, some care is needed when a naive interpretation of the seminal papers of Kinetic Theory is used in Econophysics [5]. If energy is replaced by wealth, energy elements by "elements of wealth" (money, shares, estates, coins), molecules by economic agents, it is tempting to consider a closed economy as a "perfect gas" of agents who can exchange wealth elements while conserving the total amount of wealth. We may agree with objections such as the following one: "The models... are based upon models of statistical physics in which energy is conserved in exchange processes. There are examples in economics where the principle of conservation may be 
a reasonable approximation to reality, such as primitive hunter-gatherer societies. But in the industrialised capitalist economies, income is most definitely not conserved. The process of production and not exchange is responsible for this. Models which focus purely on exchange and not on production cannot by definition offer a realistic description of the generation of income in the capitalist, industrialised economies" [6]. Nonetheless, it is useful to study conservative models, to explore their scope and limits, both for their simplicity and for the possibility of exactly solving them. Simplicity does not mean that solutions are trivial and useless, as we have shown in a previous paper [8] and has been thoroughly discussed by Aoki and Yoshikawa [9].

\section{The redistribution game}

In this series of papers, we use the word game in a wide sense, including bets and lotteries. This definition includes any stochastic direct or indirect interaction rule among agents; it could also include situations where agents selected at random interact through a payoff matrix [7], but this is not considered here. As mentioned in the Introduction, the simplest case of exchange game has been discussed in a previous papers of ours [8]. In that game, two agents (out of $g$ ) are selected by chance. One of them (the winner) receives one coin from the other agent (the loser). This procedure is then iterated. We have denoted this game as the Bennati-Dragulescu-Yakovenko (BDY) model [10-12]. As at the end of each move a coin changes agent, one could think that the second Boltzmann's method is better than the first one. However, given that the game mechanism is to extract the couple and not the coin at random, this means that the joint agent descriptions $\mathbf{Y}=\mathbf{n}:=\left(n_{1}, \ldots, n_{g}\right), \sum_{1}^{g} n_{i}=n$ are not equiprobable. Hence, we cannot simply maximize the multiplicity factor $\frac{g !}{z_{0} ! \ldots z_{n} !}$ to obtain the most probable wealth distribution $\mathbf{Z}=\left(z_{0} \ldots, z_{n}\right), \sum_{0}^{n} z_{i}=g$, $\sum_{0}^{n} i z_{i}=n$, where $z_{i}$ is the number of agents with $i$ coins, and prove that the most probable $\mathbf{Z}$ is exponential (see [8]). The correct solution is inspired to "Boltzmann I" rather than "Boltzmann II".

\subsection{Description of the game}

Here, we would like to propose another exchange game mimicking taxation and redistribution in a simplified way. We still have $g$ agents and $n$ coins. By taxation, we mean a step where coins are taken from the agents and temporarily removed from the population; by redistribution, we mean a step where coins are given back to agents. A coin is taken at random (taxation). The probability that the coin is extracted from agent $i$ is $n_{i} / n$, where $n_{i}$ is the number of coins of agent $i$ as discussed above. Then, this coin is redistributed to the agents following the rule that the $j$ th agent will receive the coin proportional to $w_{j}+n_{j}$, where $w_{j}$ is a suitable weight that will be discussed below.

If the system is in state $\mathbf{Y}_{t}=\left(n_{1}, \ldots, n_{g}\right):=\mathbf{n}$, in the subsequent step, the possible values of $\mathbf{Y}_{t+1}$ will be:
$\mathbf{Y}_{t+1}=\left(n_{1}, \ldots, n_{i}-1, \ldots, n_{j}+1, \ldots, n_{g}\right):=\mathbf{n}_{i}^{j}$, corresponding to a loss of the $i$ th agent due to taxation and a gain of the $j$ th agent due to redistribution. We propose the following transition probability between these states:

$$
P\left(\mathbf{n}_{i}^{j} \mid \mathbf{n}\right)=\frac{n_{i}}{n} \frac{w_{j}+n_{j}-\delta_{j, i}}{w+n-1}
$$

where $w=\sum_{j} w_{j}$, and the Kronecker symbol $\delta_{j, i}$ takes into account the case $j=i$. Such a simple transition probability already yields a rich and complex behaviour. Note that, with non-vanishing weights $\left\{w_{j}\right\}$, also an agent without coins can benefit from the redistribution process. In the following, we assume that $w_{j}=\alpha$ uniformly, and that $w=g \alpha=\theta$. This implies that all agents are equal with respect to the dynamics. Equation (1) defines the transition matrix of an irreducible Markov chain (all possible states $\mathbf{n}$ sooner or later communicate) which is also aperiodic. Hence, there exists an invariant probability distribution $\pi(\mathbf{n})$ that coincides with the equilibrium one. This means that $\lim _{t->\infty} P\left(\mathbf{Y}_{t}=\mathbf{n} \mid \mathbf{Y}_{0}=\mathbf{n}^{\prime}\right)=\pi(\mathbf{n})$, irrespective of the initial state $\mathbf{Y}_{0}$. Moreover, if $\alpha>0$ then $\pi(\mathbf{n})>0$ holds for all the $\left(\begin{array}{c}n+g-1 \\ n\end{array}\right)$ possible occupation numbers. The invariant distribution $\pi(\mathbf{n})$ is found to solve the detailed balance conditions, and turns out to be the $g$-variate Polya distribution [13]

$$
\pi(\mathbf{n} ; \alpha, g)=\frac{n !}{\theta^{[n]}} \prod_{i=1}^{g} \frac{\alpha^{\left[n_{i}\right]}}{n_{i} !}
$$

where $x^{[n]}=x(x+1) \ldots(x+n-1)$ is the Pochhammer symbol. Well-known subcases of (2) are:

$-\pi(\mathbf{n})=\left(\begin{array}{c}n+g-1 \\ n\end{array}\right)^{-1}$ uniform on all $\mathbf{n}$, for $\alpha=1$

- the multinomial distribution $\pi(\mathbf{n})=\frac{n !}{\prod_{i=1}^{g} n_{i} !} g^{-n}$, for $\alpha \rightarrow \infty$

- and finally $\pi(\mathbf{n})=\left(\begin{array}{l}g \\ n\end{array}\right)^{-1}$ uniform on the restricted support of all $\mathbf{n}$ with $n_{i}=0,1$, and obtained for $\alpha=-1$.

In other words, $\alpha=1$ gives the Bose-Einstein distribution; $\alpha \rightarrow \infty$ the Maxwell-Boltzmann distribution, and $\alpha=-1$ the Fermi-Dirac distribution [13]. The redistribution policy is then determined by the value of $\alpha$. Positive values make rich agents richer, and this effect is larger the smaller is $\alpha ; \alpha \rightarrow \infty$ determines a redistribution where all agents are equivalent, irrespective of their wealth; finally, negative values of $\alpha$ tend to favour poor agents. In the latter case, it is possible to show that $|\alpha|$ is the maximum allowed value of wealth for each agent.

\subsection{Block taxation}

Let us now suppose that taxation is made in a block, instead of extracting a single coin from one agent for each step. With this mechanism, a fraction of the wealth is 
taken, and then redistributed and equation (1) has to be generalized to $m \leq n$ coins.

Let $\mathbf{m}=\left(m_{1}, \ldots, m_{g}\right)$, with $\sum_{1}^{g} m_{i}=m$, be the taxation vector, whereas let $\mathbf{m}^{\prime}=\left(m_{1}^{\prime}, \ldots, m_{g}^{\prime}\right)$, with $\sum_{1}^{g} m_{i}^{\prime}=m$, be the redistribution vector.

Now the new state becomes $\mathbf{n}^{\prime}=\mathbf{n}-\mathbf{m}+\mathbf{m}^{\prime}$, but the resulting chain still has (2) as equilibrium distribution. The only difference between the one-coin taxationredistribution mechanism and the $m$-coin one is the rate of approach to equilibrium: an increasing function of $m$, i.e. $r=\frac{m \theta}{n(\theta+n-m)}$. The inverse of $r$ is roughly the number of transitions needed to reach equilibrium. Notice that if $m=n$, that is all the wealth is taxed and redistributed, the new state has no memory of the previous one, and the probability distribution is given by equation (2) only after one step $(r=1)$.

\subsection{Marginal description}

We have defined a model of taxation-and-redistribution. The model can be exactly solved with respect to the individual agent descriptions. Deriving the wealth distribution is formally easy, as all the agent descriptions can be divided into equivalence classes considering only the numbers (and not the names) of the agents with $i$ coins, $i=0,1, \ldots, n$. Since two agent descriptions $\left\{\ldots, Y_{i}=\right.$ $\left.a, \ldots, Y_{j}=b, ..\right\}$ and $\left\{\ldots, Y_{i}=b, \ldots, Y_{j}=a, ..\right\}$ have the same probability at equilibrium, then $\Pi(\mathbf{z})=\frac{g !}{\prod_{0}^{n} z_{i} !} \pi(\mathbf{n})$, where $z_{i}$ is the number of agents with $i$ coins. However, $\pi(\mathbf{n})$ is not uniform except in the case $\alpha=1$. Hence, the usual constrained maximization that would give the most probable vector $\mathbf{z}^{*}$ cannot be performed. This difficulty leads us to use the marginal method discussed in the first Boltzmann's paper discussed in the introduction. That method is exact and it was later abandoned after the success of the approximate maximization method. Let us consider an agent, say $i=1$ and let us consider the transition probability (1) projected onto two categories: the first category includes agent number 1 and the second category all the remaining agents. As all the agents are equivalent, the index 1 can be omitted. Let us start with $Y_{t}=k$. Then defining $\lambda_{k}:=P\left(Y_{t+1}=k+1 \mid Y_{t}=k\right)$, and $\mu_{k}:=P\left(Y_{t+1}=k-1 \mid Y_{t}=k\right)$, we get a simple birth-death chain, with $\lambda_{k}=\frac{n-k}{n} \frac{\alpha+k}{\theta+n-1} ; \mu_{k}=\frac{k}{n} \frac{\theta-\alpha+n-k}{\theta+n-1}$, that is a random walk in $\{0,1, \ldots, n\}$ with semi-reflecting barriers. For this chain, the equilibrium distribution exists, and it is

$$
\pi(k)=\frac{n !}{k !(n-k) !} \frac{\alpha^{[k]}(\theta-\alpha)^{[n-k]}}{\theta^{[n]}} ;
$$

this equation defines the bivariate Polya distribution with weights $\alpha$ and $\theta-\alpha$. Then the average value of the random variable $k$ is $E(k)=n \frac{\alpha}{\theta}=\frac{n}{g}$ and its variance is $\operatorname{Var}(k)=$ $n \frac{\alpha}{\theta} \frac{\theta-\alpha}{\theta} \frac{\theta+n}{\theta+1}=n \frac{1}{g} \frac{g-1}{g} \frac{\theta+n}{\theta+1}$.

If $\alpha \rightarrow \infty, \frac{\alpha}{\theta}=\frac{1}{g}$, and $\pi(k)$ tends to the $\operatorname{Binomial}\left(n, \frac{1}{g}\right)$.
If $\alpha<0$, the ascending factorial (Pochhammer symbol) becomes $(-x)^{[n]}=(-x)(-x+1) \ldots(-x+n-1)=$ $(-1)^{n}|x|(|x|-1) \ldots(|x|-n+1):=(-1)^{n}|x|_{[n]}$, hence

$$
\frac{\alpha^{[k]}(\theta-\alpha)^{[n-k]}}{\theta^{[n]}}=\frac{|\alpha|_{[k]}(|\theta-\alpha|)_{[n-k]}}{(|\theta|)_{[n]}},
$$

and for $|\alpha|$ integer (3) becomes $\frac{\left(\begin{array}{c}|\alpha| \\ k\end{array}\right)\left(\begin{array}{c}|\theta-\alpha| \\ n-k\end{array}\right)}{\left(\begin{array}{c}|\theta| \\ n\end{array}\right)}$, that

is the Hypergeometric $(n,|\alpha|,(g-1)|\alpha|)$. In this case the range of the random walk is $\{0,1, \ldots, \min (|\alpha|, n)\}$.

It is interesting to remark that the three forms of the equilibrium distribution have the same average $E(k)=\frac{n}{g}$, but very different variance, according to the sign and the size of $\theta \cdot \operatorname{Var}(k) \approx n^{2}$ for $\theta$ positive and not too large, $\operatorname{Var}(k)=n \frac{1}{g} \frac{g-1}{g} \approx n$ for the independent redistribution $\alpha \rightarrow \infty$, and, finally, for $\alpha<0, \operatorname{Var}(k)=n \frac{1}{g} \frac{g-1}{g} \frac{|\theta|-n}{|\theta|-1}$ which is even smaller.

\subsection{Marginal description and block taxation}

In the block case, the marginal chain is much more complicated than a simple birth-death chain, because at each step $|\Delta k|$ can vary from 0 to $m$. Then $Y_{t+1}=Y_{t}-D_{t+1}+$ $C_{t+1}=Y_{t}+I_{t+1}$, where $D_{t+1}$ is the random taxation for the fixed agent and $C_{t+1}$ is its random redistribution. If we study only the first moment, $E\left(D_{t+1} \mid Y_{t}=k\right)=m \frac{k}{n}$, and $E\left(C_{t+1} \mid Y_{t}=k, D_{t+1}=d\right)=m \frac{\alpha+k-m \frac{k}{n}}{\theta+n-m}$, therefore

$$
E\left(\Delta k_{t+1} \mid k_{t}\right)=-\frac{m \theta}{n(\theta+n-m)}\left(k_{t}-n \frac{\alpha}{\theta}\right) .
$$

The following remarks can be made:

- if $k_{t}=n \frac{\alpha}{\theta}$, the chain is first-order stationary;

- the equation is analogous to a restoring force around the expected value $E(k)=n \frac{\alpha}{\theta}=\frac{n}{g}$;

- the intensity of the restoring force is $r=\frac{m \theta}{n(\theta+n-m)}$. As a function of the total weight $\theta$, it is $O(\theta)$ for small $\theta$, in which case the rate is very slow, and an agent can stay for a long time very far from the expected wealth $\frac{n}{g}$. If $\theta \rightarrow \infty$, the equilibrium approach rate is $\frac{m}{n}$, and the rate increases if $\theta<0$.

In the case $\theta<0$, there is a maximum allowed wealth. A consistency condition for the model, excluding transient states, is necessary. For instance, if the maximum allowed wealth is ten times the average, $|\alpha|=10 \frac{n}{g}$, thus $|\theta|=10 n$, $r=\frac{10 m n}{n(10 n-n+m)}=\frac{10 m}{10 n-n+m} \simeq \frac{10}{9} \frac{m}{n}$ if $m \ll$ $n$, not so far from the independent redistribution case. If $|\alpha|=3 \frac{n}{g}$, then one has $r=\frac{3 m}{3 n-n+m} \simeq \frac{3}{2} \frac{m}{n}$. The extreme case is $|\alpha|=\frac{n}{g}$, leading to $r=\frac{m}{n-n+m}=1$. In this case, the state vector is $\mathbf{Y}(t)=(n / g, \ldots, n / g)$ 
for sure, and the redistribution deterministically produces the same vector. In other words, one trivially has $r=1$. If we allow an initial state containing individuals richer than $|\alpha|$, that is if we consider (4) for $k_{t}>|\alpha|$, then $E\left(D_{t+1} \mid Y_{t}=k\right)$ is still $m \frac{k}{n}$, but $E\left(C_{t+1} \mid Y_{t}=k, D_{t+1}=\right.$ $d)=0$ unless $k-d<|\alpha|$. More precisely, one has

$$
E\left(C_{t+1} \mid k, d\right)=\left\{\begin{array}{c}
m \frac{|\alpha|-k+m \frac{k}{n}}{|\theta|-n+m} \text { if } k-m \frac{k}{n} \leq|\alpha| \\
0 \text { if } k-m \frac{k}{n}>|\alpha|
\end{array} .\right.
$$

Letting the average percent taxation be $f=\frac{m}{n}$, then one gets

$$
E\left(\Delta k_{t+1} \mid k_{t}\right)=\left\{\begin{array}{c}
-\frac{f \theta}{\theta-n(1-f)}\left(k_{t}-\frac{n}{g}\right) \text { if } k(1-f) \leq|\alpha| \\
-k(1-f) \text { if } k(1-f)>|\alpha|
\end{array} .\right.
$$

As $k(1-f)$ is the average value after taxation, even if the agent is initially richer than $|\alpha|$ he/she can participate to redistribution, if the mean percentage of taxation is high enough.

\subsection{The mean wealth distribution}

The actual wealth distribution $\mathbf{Z}=\left(z_{0} \ldots, z_{n}\right), \sum_{0}^{n} z_{i}=$ $g, \sum_{0}^{n} i z_{i}=n$, where, as written before, $z_{i}$ is the number of agents with $i$ coins, is a random variable moving in time according to the dynamics (1) or, more realistically, to an $m$ - block taxation-and-redistribution mechanism. The random variable $\mathbf{Z}$ is a $(n+1)$-dimensional vector, where $n$ can be very large, and the domain of $\mathbf{Z}$, fixed by the two constraints, is at least as large as nearly $\frac{1}{4 \sqrt{3} n} \exp \left[\pi \sqrt{\frac{2 n}{3}}\right]$. Given $\mathbf{Z}$, the probability that a randomly extracted agent is $i$-rich is $\frac{z_{i}}{g}$. As said before, we can study the link between "Boltzmann I" and "Boltzmann II", that is between the marginal distribution of agent wealth and the wealth distribution of the whole population. Indeed, we have $g$ variables $Y_{1}, \ldots, Y_{g}$, with the same probability distribution; therefore, the expected value of the number of agents with $i$ coins is $E\left(z_{i}\right)=P\left(Y_{1}=i\right)+\ldots+P\left(Y_{g}=i\right)=g P_{i}$, where $P_{i}$ is given by equation (3) (see Appendix). If "Boltzmann II" works, one finds $\mathbf{Z}^{*}$ - the most probable frequency vector - supposed to coincide with the expected value $E(\mathbf{Z})$, wherefrom one derives $P_{i}=\frac{E\left(z_{i}\right)}{g}$. In this paper, we have applied "Boltzmann I", so that we obtain $E(\mathbf{Z})$ in terms of the marginal distribution $P_{i}$. The exact probabilistic meaning of the loose expression "equilibrium wealth distribution" is twofold: $P_{i}$ is the expected fraction of agents whose wealth is $i$, or the probability that any fixed agent is $i$-rich, when equilibrium is reached.

\subsection{Thermodynamic limit}

It is useful to consider the thermodynamic limit $g \gg$ $1, n \gg 1, n / g=\alpha \chi$. The name is suggested by the fact that under these conditions, equation (3) describes the interaction of a fixed agent with the very large thermostat made up of all the other agents. Starting from $P_{k}=\pi(k)=\frac{n !}{k !(n-k) !} \frac{\alpha^{[k]}(\theta-\alpha)^{[n-k]}}{\theta^{[n]}}$, and denoting the thermodynamic limit by $T L$, one gets

$$
P_{k}^{T L}=\frac{\alpha^{[k]}}{k !}\left(\frac{1}{1+\chi}\right)^{\alpha}\left(\frac{\chi}{1+\chi}\right)^{k}
$$

that is the Negative Binomial $(\alpha, \chi)[2]$.

The moments of the Polya distribution and the corresponding ones of the limiting NegativeBinomial $(\alpha, \chi)$ are

$$
\begin{aligned}
E(k) & =n \frac{\alpha}{\theta} \rightarrow E^{T L}(k)=\alpha \chi \\
\operatorname{Var}(k) & =n \frac{1}{g} \frac{g-1}{g} \frac{\theta+n}{\theta+1} \rightarrow \operatorname{Var}^{T L}(k)=\alpha \chi(1+\chi) .
\end{aligned}
$$

Note that, here, the wealth domain is $\{0,1,2, \ldots, \infty\}$. We can now consider the continuum limit $T L C$, given that for $\chi \gg 1$ the Negative Binomial tends to the Gamma Distribution. Indeed, for $\chi \gg 1$ and $\alpha$ finite the expected number of coins is large, and we can pass to a continuous random variable without danger:

$$
\begin{aligned}
P_{k}^{T L} & =\frac{\alpha^{[k]}}{k !}\left(\frac{1}{1+\chi}\right)^{\alpha}\left(\frac{\chi}{1+\chi}\right)^{k} \\
& \approx \frac{k^{\alpha-1}}{\Gamma(\alpha)}\left(\frac{1}{\chi}\right)^{\alpha}\left(1-\chi^{-1}\right)^{k} \approx \frac{k^{\alpha-1}}{\chi^{\alpha} \Gamma(\alpha)} \exp \left[-\frac{k}{\chi}\right]
\end{aligned}
$$

that is $\operatorname{Gamma}(\alpha, \chi)$.

More precisely, $P(k) \simeq \int_{k-1 / 2}^{k+1 / 2} \operatorname{Gamma}(x ; \alpha, \chi) d x$.

The average value is conserved, i.e. $E^{T L C}(k)=\alpha \chi$, the variance becomes $\operatorname{Var}^{T L C}(k)=\alpha \chi^{2}$, and the kurtosis excess is $\frac{6}{\alpha}$.

Then our simple exact model leads to the Gamma distribution, which is actually very popular as an income distribution. In this model the form factor $\alpha$ is essential, as most concentration indices are independent from the scale factor $\chi$. For instance Gini's coefficient is $R(\alpha)=\frac{\Gamma(2 \alpha+1)}{\left(2^{\alpha} \Gamma(\alpha+1)\right)^{2}}=\frac{(2 \alpha) !}{4^{\alpha} \alpha !^{2}}$ for $\alpha$ integer, a decreasing function of $\alpha$. Note that for $\alpha=1.5$ we have $R=0.42$ and for $\alpha=2.5$ we have $R=0.34$. These are realistic bounds for many industrialised economies [14].

\section{Discussion and conclusions}

As mentioned before, the Gamma model is very popular as an income distribution law. Income should be not confused with wealth. Income is a flux, whereas wealth is a stock. In 1898, March [15] proposed the Gamma probability density function (pdf) to fit the distribution of wages in France, Germany and the United States. In 1925, Amoroso [16] derived and applied a generalized Gamma pdf to income distribution in Prussia. In more recent years, Salem and Mount [17] fitted the Gamma pdf to the income data 
in United Stated. The Gamma model is a member of D'Addario's system [18] which formally corresponds to the Brillouin's statistics. Angle derives the Gamma distribution as the limiting distribution of his inequality process [19].

Coming back to our model, one has an interesting interpretation for the form factor $\alpha$ of the Gamma distribution as the initial weight for the redistribution mechanism. Our taxation-and-redistribution (TAR) game cannot be taken too seriously, because it is played considering all other economic activities as completely frozen. In an economic system, many other "games" occur at the same time, even if the total amount of wealth is constant within the period of investigation. One can think of a system where both the BDY game and the TAR game take place. Suppose that at times $t_{1}, t_{1}+1, \ldots, t_{1}+m-1$, the BDY game is played, and, at times $t_{2}=t_{1}+m, t_{2}+1, \ldots, t_{2}+n-$ $1, n$ moves of the TAR game are made. The BDY game shifts the initial wealth distribution towards an exponential, and such a distribution becomes the initial one for the TAR game. Then, the TAR game shifts the distribution towards a Gamma and this process can be iterated many times. We would need the relaxation times for each game, which actually are much greater than the intervals $m, n$ where each of them is supposed to be played without interruption, for a complete description of the mixed process. We can guess that the equilibrium distribution of the joint process will be a mixture of the two "pure" ones, with weights proportional to the intensity and efficiency of the two processes.

In the case of both BDY and TAR, computer simulations are not necessary, because the equilibrium distributions can be analytically derived. In contrast, computer simulations become necessary when these games are played sequentially or at the same time. This will be a topic for future research. But, before doing that, it will be useful to introduce a third type of game: the Zipf-SimonYule (ZSY) one, where the number of agents is not conserved, but the total amount of coins does not change. This mechanism produces Paretian power-law tails, if the redistribution mechanism is no longer Polya, but à la Zipf [20]. This game can be analytically solved as well [21]. The interplay among the BDY game, the TAR game and the ZSY one may give a satisfactory representation of a conservative economic system where total wealth does not change (but see [22] for a discussion on the non-conservation of money).

It is interesting to compare the discrete models of this paper and of reference [8] with the exchange models by Angle [19] and by Chakraborti-Chakrabarti [23]. In our case, following Boltzmann, we use a totally discrete approach and then derive continum limits, whereas in those papers, the authors consider the wealth as a continous random variable.

Finally, with respect to the problem of agent interactions, it is possible to consider them on a complex graph (or network) [24] instead of on a random graph.
Authors are indebted to D. Costantini and L. Di Gennaro for helpful discussions.

\section{Appendix}

(1) Given $\mathbf{Z}=z$, the probability that an agent extracted at random at time $t$ is $i$-rich is $\frac{z_{i}}{g}$. Hence $P\left(A_{t}=i \mid\right.$ $\left.\mathbf{z}_{t}, \mathbf{y}_{0}\right)=\frac{z_{i}}{g}$ is universal, it is independent from the initial state $\mathbf{y}_{0}$ and it holds also far from equilibrium. Note that we do not know in advance the name of the extracted agent. Let us instead consider a fixed agent $j$, whose initial wealth was $Y_{j}(0)=k \gg i$. In general $P\left(Y_{j}(t)=i \mid\right.$ $\left.\mathbf{z}, Y_{j}(0)=k\right) \neq \frac{z_{i}}{g}$, because if $t$ is not so large the memory of the past wealth is still important. Also, if we do not exactly know $\mathbf{Z}, P\left(A_{t}=i\right)=\sum_{\mathbf{z}} P\left(A_{t}=i \mid \mathbf{z}(t)\right) P(\mathbf{z})=\frac{E\left(z_{i}(t)\right)}{g}$ only holds for a randomly extracted agent. Note that $E\left(z_{i}(t)\right)$ is still a function of the initial conditions.

The random variable $Z_{i}$ counts the number of agents whose wealth is $i$, then $Z_{i}=\sum_{j=1}^{g} 1_{Y_{j}=i}$, where $1_{Y_{j}=i}=1$ if $Y_{j}=i, \quad$ or $1_{Y_{j}=i}=0$ if $Y_{j} \neq i$. Then $E\left[Z_{i}\right]=$ $E\left[\sum_{j=1}^{g} 1_{Y_{j}=i}\right]=\sum_{j=1}^{g} E\left[1_{Y_{j}=i}\right]=\sum_{j=1}^{g} P\left(Y_{j}=i\right)$. This is true at any time, $t, Z_{i}(t)=\sum_{j=1}^{g} 1_{Y_{j}(t)=i}$, hence

$$
E\left[Z_{i}(t)\right]=\sum_{j=1}^{g} P\left(Y_{j}(t)=i\right)
$$

For $t \rightarrow \infty, E\left[Z_{i}(\infty)\right]=\sum_{j=1}^{g} P\left(Y_{j}(\infty)=i\right)=g P_{i}$. Then $P\left(Y_{j}(\infty)=i\right)=P_{i}=\frac{E\left(z_{i}(\infty)\right)}{g}$ holds for all agents, as they are going to be described by the same probability distribution as time goes by and the memory of initial conditions is lost.

(2) Suppose that starting from a system with $n$ coins (whose value is 1 ) in the thermodynamic limit $n, g \gg 1$, where $\alpha \chi=\frac{n}{g}$. Then $P_{k}^{T L}=\frac{\alpha^{[k]}}{k !}\left(\frac{1}{1+\chi}\right)^{\alpha}\left(\frac{\chi}{1+\chi}\right)^{k}$ is the probability that an agent $X$ is $k$-rich. Now we compare this system with a new one, where we change any coin (whose value is 1 ) with $m$ coins whose value is $\frac{1}{m}$. If the dynamics is the same (that is $\alpha$ is the same), the equilibrium probability that an agent $Y$ is $k$-rich is now $P_{k^{\prime}}^{T L}=$ $\frac{\alpha^{\left[k^{\prime}\right]}}{k^{\prime} !}\left(\frac{1}{1+\chi^{\prime}}\right)^{\alpha}\left(\frac{\chi^{\prime}}{1+\chi^{\prime}}\right)^{k^{\prime}}$, where $k^{\prime}=m k, \chi^{\prime}=m \chi$. The probability in the initial system $P\{X \in[k, k+1)\}$ corresponds to the probability (in the new system) $P\{Y \in$ $[m k, m k+m)\} \simeq m P\{Y=m k)=m P_{k^{\prime}}^{T L}$.

$$
\operatorname{Now} P_{k^{\prime}}^{T L}=P_{m k}^{T L}=\frac{\alpha^{[m k]}}{(m k) !}\left(\frac{1}{1+m \chi}\right)^{\alpha}\left(\frac{m \chi}{1+m \chi}\right)^{m k}
$$


and if $m$ is large:

$$
\begin{aligned}
& P_{k^{\prime}}^{T L}=\frac{\alpha^{[m k]}}{(m k) !}\left(\frac{1}{1+m \chi}\right)^{\alpha}\left(\frac{m \chi}{1+m \chi}\right)^{m k} \\
& \simeq \frac{(m k)^{\alpha-1}}{\Gamma(\alpha)}\left(\frac{1}{m \chi}\right)^{\alpha}\left(1-\frac{1}{m \chi}\right)^{m k} \\
& \simeq \frac{1}{m} \frac{k^{\alpha-1}}{\Gamma(\alpha)}\left(\frac{1}{\chi}\right)^{\alpha} \exp \left[-\frac{k}{\chi}\right], \text { that is : } \\
& \lim _{m \rightarrow \infty}[m P\{Y=m k)]=\operatorname{Gamma}[\alpha, \chi] .
\end{aligned}
$$

Hence: if the initial system was already in the condition $P\{X \in[k, k+1)\} \simeq \operatorname{Gamma}[\alpha, \chi]$, this is not affected by the change of numeraire, but now the distribution has domain of support $[0, \infty)$, so that one can exchange fractions of the unit of wealth.

\section{References}

1. L. Boltzmann, Wissenschaftliche Abhandlungen, edited by Hasenöhrl (1909), Vol. 1, pp. 49-96

2. E. Parzen, Modern Probability Theory and Its Applications (Wiley, 1960)

3. L. Boltzmann, Wissenschaftliche Abhandlungen, edited by Hasenöhrl (1909), Vol. 2., pp. 164-223

4. A. Bach, Arch. Ex. Sci. 41, 1 (1990)

5. A. Chatterjee, S. Yarlagadda, B.K. Chakrabarti, Econophysics of Wealth Distributions (Springer, Berlin, 2005)

6. M. Gallegati, S. Keen, T. Lux, P. Ormerod, Physica A 370, 1 (2006)
7. R. Axtell, J.M. Epstein, H.P. Young, The Emergence of Classes in a Multi-Agent Bargaining Model, Working Paper No. 9, Center on Social and Economic Dynamics, February 2000

8. E. Scalas, U. Garibaldi, S. Donadio, Eur. Phys. J. B 53, 267 (2006)

9. M. Aoki, H. Yoshikawa, Reconstructing Macroeconomics: A Perspective from Statistical Physics and Combinatorial Stochastic Processes (Cambridge University Press, Cambridge, UK, 2006)

10. E. Bennati, Riv. Int. Sci. Econom. Comme. 35, 735 (1988)

11. A. Dragulescu, V.M. Yakovenko, Eur. Phys. J. B 17, 723 (2000)

12. V.M. Yakovenko, Statistical Mechanics Approach to Econophysics, Springer Encyclopedia of Complexity and System Science http://ref works . springer . com/complexity/

13. D. Costantini, U. Garibaldi, Found. Phys. 30, 81 (2000)

14. http://en.wikipedia.org/wiki/Gini_coefficient

15. L. March, J. Soc. Stat. Paris 193, 241 (1898)

16. L. Amoroso, Ann. Mat. Pura Appl. 21, 123 (1925)

17. A.B. Salem, T.D. Mount, Econometrica 42, 1115 (1974)

18. R. D'Addario, Giorn. Econ. Ann. Econ. 8, 91 (1949)

19. J. Angle, Physica A 367, 388 (2006)

20. U. Garibaldi, D. Costantini, S. Donadio, P. Viarengo, Physica A 355, 224 (2005)

21. U. Garibaldi, D. Costantini, S. Donadio, P. Viarengo, Comput. Econ. 27, 115 (2006)

22. S. Keen, working paper, 2007

23. A. Chakraborti, B.K. Chakrabarti, Eur. Phys. J. B 17, 167 (2000)

24. T. Di Matteo, T. Aste, S.T. Hyde, in The Physics of Complex Systems, edited by F. Mallamace, H.E. Stanley, Proceedings of International School of Physics "Enrico Fermi" (IOS Press, Amsterdam, 2004), pp. 435-442 